\title{
Absorption spectroscopy of trapped rubidium atoms
}

\author{
S. Cauchi, A. Vorozcovs, M. Weel, S. Beattie, O. Gagnon, and \\ A. Kumarakrishnan
}

\begin{abstract}
We determine the absolute density of a sample of laser-cooled atoms in a twolevel system by recording the absorption spectrum of the ${ }^{85} \mathrm{Rb}_{5 \mathrm{~S}_{1 / 2}}\left(F=3, m_{\mathrm{f}}=3\right) \rightarrow$ $5 \mathrm{P}_{3 / 2}\left(F^{\prime}=4, m_{\mathrm{f}}^{\prime}=4\right)$ transition. Trapped atoms were prepared in the $(F=3$, $m_{\mathrm{f}}=3$ ) ground state through optical-pumping techniques. We compare our results with an independent measure of the density that relies on a direct measurement of the number of atoms and size of the atomic sample. We also study the contributions of power broadening, laser line width, and Doppler broadening to the measured absorption spectrum. Our studies suggest that the natural line width $(\sim 6 \mathrm{MHz})$ can be measured to a precision of less than $\sim 50 \mathrm{kHz}$ if the laser line width is measured in real-time with a high-finesse Fabry-Perot cavity.
\end{abstract}

PACS Nos.: 32.70.Cs, 32.70.Jz, 32.80.Pj, 42.62.Fi, 32.70.-n, 32.30.-r

Résumé : Nous avons déterminé la densité absolue d'un échantillon d'atomes refroidis par laser dans un système à deux niveaux, en enregistrant le spectre d'absorption de la transition $5 \mathrm{~S}_{1 / 2}\left(F=3, m_{\mathrm{f}}=3\right) \rightarrow 5 \mathrm{P}_{3 / 2}\left(F^{\prime}=4, m_{\mathrm{f}}^{\prime}=4\right)$ dans le ${ }^{85} \mathrm{Rb}$. Les atomes dans le piège sont préparés dans le fondamental $\left(F=3, m_{\mathrm{f}}=3\right)$ à l'aide d'une technique de pompage optique. Nous comparons nos résultats avec une mesure indépendante de la densité qui repose sur la mesure directe du nombre d'atomes et de la dimension de l'échantillon d'atomes. Nous avons aussi étudié la contribution de l'élargissement en puissance, de la largeur de ligne et de l'élargissement Doppler du spectre d'absorption mesuré. Nos résultats suggèrent que la largeur naturelle de ligne $(\approx 6 \mathrm{MHz})$ peut être mesurée avec une précision meilleure que $50 \mathrm{MHz}$ si la largeur de ligne laser est mesurée en temps réel avec une cavité Fabry-Perot de haute précision.

[Traduit par la Rédaction]

\section{Introduction}

During the last fifteen years, significant improvements in precision measurements have been achieved using cold samples of neutral atoms in magneto-optical traps $[1,2]$. Such measurements include the

Received 20 August 2003. Accepted 17 August 2004. Published on the NRC Research Press Web site at http://cjp.nrc.ca/ on 17 November 2004.

S. Cauchi, ${ }^{1}$ A. Vorozcovs, M. Weel, S. Beattie, O. Gagnon, and A. Kumarakrishnan. ${ }^{2}$ Department of Physics, York University, 4700 Keele Street, Toronto, ON M3J 1P3, Canada.

${ }^{1}$ Present Address: Edward S. Rogers Department of Electrical and Computer Engineering, University of Toronto, 10 King's College Road, Toronto, ON M5S 3G4, Canada.

${ }^{2}$ Corresponding author (e-mail: akumar@yorku.ca). 
determination of gravitational acceleration and rotation using atom interferometry $[3,4]$, the atomic fine structure constant [5], and atomic level structure [6].

In addition, one of the best known measurements of the natural line width was carried out in laser-cooled sodium atoms using a stabilized laser with a line width of a few $\mathrm{kHz}$ [7]. Measurements with comparable precision have also been obtained using photo-associative spectroscopy of ultra-cold molecules [8-10]. Atom traps have also been used to improve the accuracy of atomic clocks [11] and study cold collisions [12]. For many of these applications, it is necessary to understand the scaling laws that govern the density distribution and temperature of cold atoms [13,14]. This involves the use of reliable diagnostic techniques such as the determination of the number of atoms by fluorescence detection using a photomultiplier tube (PMT) [15], cloud size and temperature using charge-coupled devices (CCD) [16,17], and density using absorption spectroscopy [18].

In this work, we compare the absolute density determined using two techniques. The first measurement involves determining the number of atoms and the volume of the sample using a PMT and two CCDs, respectively. An independent method involves turning off the confining forces and scanning a weak probe laser across the atomic resonance and recording the absorption spectrum.

The natural line width, which can be inferred from the absorption spectrum, has Lorentzian contributions due to the laser line width and power broadening. The spectrum also has a residual Gaussian contribution due to Doppler broadening. In this work, we measure the effect of power broadening by recording the spectrum as a function of probe power. By observing the expansion of the atomic cloud with CCDs, we measure the velocity distribution and infer the Doppler contribution. Our results indicate that it should be possible to measure the natural line width to a precision of $<1 \%$ if the laser line width can be measured using a high-finesse Fabry-Perot cavity [19].

The motivation for renewed interest in line-width measurements are related to tests of improved calculations of atomic structure. In recent work [7], the natural line width of $\mathrm{Na}$ atoms ( $\sim 10 \mathrm{MHz})$ was measured using an atom trap to a precision of $22 \mathrm{kHz}$ using a cavity-stabilized laser with a line width of $1.5 \pm 3 \mathrm{kHz}$. In this case, a weak probe laser was scanned across the resonance of a velocity-selected group of atoms and the resulting fluorescence was observed. In ref. 20, the radiative decay time of the $5 \mathrm{P}_{3 / 2}$ and $5 \mathrm{P}_{1 / 2}$ states in trapped ${ }^{87} \mathrm{Rb}$ were measured to a precision of $<0.25 \%$ after excitation by a short, resonant pulse. Fast beam techniques have resulted in comparable precision in ${ }^{87} \mathrm{Rb}$ [21], whereas, the earlier Hanle spectroscopy measurement [22] seems to be the most precise in ${ }^{85} \mathrm{Rb}$.

As noted in ref. 20, the Doppler contribution in rubidium is much smaller than in sodium. This implies that precision comparable to that of ref. 20 can be achieved with cold atoms without the need for the same degree of velocity selection. Our results suggest that a precision measurement is possible with reduced experimental complexity if the temperature of the sample and the laser line width can be precisely measured. It would also be relatively straightforward to extend our measurements to the ${ }^{85} \mathrm{Rb}$ $5 \mathrm{~S}_{1 / 2}(F=3) \rightarrow 5 \mathrm{P}_{3 / 2}\left(F^{\prime}=4\right)$ transition and the principal resonance lines in ${ }^{87} \mathrm{Rb}$.

The rest of this paper is divided into the following sections. In Sect. 2, we discuss the theory related to the absorption spectrum and the measurement of the density distribution using two techniques. In Sect. 3, we describe experimental details, and in Sect. 4, we discuss our results and anticipated precision.

\section{Background}

The number of atoms in the excited state, $N_{\mathrm{exc}}$, is given by

$N_{\text {exc }}=f N$

where $f$ is the fraction of atoms in the excited state, and $N$ is the total number of atoms in both the ground and excited states. This fraction is given by

$f=\frac{I / I_{\mathrm{SAT}}}{1+2 I / I_{\mathrm{SAT}}+(2 \Delta / \Gamma)^{2}}$ 
Here, $\Delta$ is the detuning of the trap laser from resonance, $I_{S A T}$ is the saturation intensity defined by $I / I_{\mathrm{SAT}}=\Omega_{\mathrm{R}}^{2} / \Gamma^{2} . \Gamma$ is the natural line width of the trapping transition and $\Omega_{\mathrm{R}}$ is the Rabi frequency. $I$ is the sum of the peak intensities of all trapping beams, and is evaluated by measuring the total power and the spatial profile of the beams. In the case of isotropic fluorescence, $N$ is given by

$N=\frac{4 \pi}{\Omega} \frac{S}{f E \Gamma}$

$N$ can be determined by measuring the power recorded by the PMT, $S$; the solid angle subtended by the detection system, $\Omega$; knowledge of the excited state fraction, $f$; and the photon energy, $E$.

The spatial distribution of the atomic cloud can be determined by fitting a CCD image to a Gaussian profile given by

$n(\boldsymbol{r})=n_{0} \cdot \exp \left\{-2\left[\left(\frac{x}{x_{0}}\right)^{2}+\left(\frac{y}{y_{0}}\right)^{2}+\left(\frac{z}{z_{0}}\right)^{2}\right]\right\}$

where $n_{0}$ is the peak density of the atomic cloud, and $x_{0}, y_{0}$, and $z_{0}$ are the $1 / e^{2}$ radii of the atomic cloud. Since $N$ and $n(\boldsymbol{r})$ are related by

$N=\iiint n(\boldsymbol{r}) \mathrm{d} V$

the above integral can be evaluated to relate the peak density of the atomic cloud to the total number of atoms and the dimensions of the cloud. This can be expressed by

$n_{0}=\frac{N}{x_{0} y_{0} z_{0}}\left(\sqrt{\frac{2}{\pi}}\right)^{3}$

Thus, this measurement of $n_{0}$ relies on a PMT for measuring a fraction of the isotropic fluroescence emitted into a known solid angle and two orthogonal CCDs for determining the dimensions of the atomic cloud.

Alternatively, we can measure the peak density of the atomic sample by allowing the atoms to decay to the ground state and measuring the absorption of a weak probe laser that is scanned across the atomic resonance. The absorption of the probe beam (propagating along the $x$-direction) through a cold gas of atoms is given by [23]

$I=I_{0} \cdot \exp \left[-\int_{-\infty}^{\infty} \alpha(\omega, x) \mathrm{d} x\right]$

where $I$ is the emerging laser intensity, $I_{0}$ is the incident laser intensity, and $\alpha(\omega, x)$ is the frequencydependent absorption coefficient. For trapped atoms with a Gaussian spatial distribution, $\alpha$ can be approximated by a Lorentzian function centered at the resonant frequency, $\omega_{0}$, as

$\alpha(\omega, x)=K \frac{f_{i k} \Gamma_{\text {eff }} n_{0} * \exp \left[-2\left(x / x_{0}\right)^{2}\right]}{\left(\omega-\omega_{0}\right)^{2}+\left(\Gamma_{\text {eff }} / 2\right)^{2}}$

Here, $\Gamma_{\text {eff }}$ is the effective line width, $n_{0}$ is the peak density of the distribution along the direction of probe, $K$ can be written in terms of the electron charge, the electron mass, the speed of light, and the permittivity of free space as

$K=\frac{e^{2}}{4 \pi \epsilon_{0} m c}$ 
and $f_{i k}$ is the oscillator strength of the atomic transition for a two-level system, given by

$f_{i k}=\frac{\epsilon_{0} m c^{3}}{2 \pi e^{2} v^{2}}$

Using (8) in (7), we obtain

$I=I_{0} \cdot \exp \left[-K \frac{\left(x_{0} \sqrt{\frac{\pi}{2}}\right) f_{i k} \Gamma_{\text {eff }} n_{0}}{\left(\omega-\omega_{0}\right)^{2}+\left(\Gamma_{\text {eff }} / 2\right)^{2}}\right]$

It is possible to fit the absorption spectrum to (11), if the size of the cloud along the direction of the probe, $x_{0}$, is determined from a CCD measurement. This results in an independent measure of the peak density, $n_{0}$.

For a precision measurement of the natural line width, the dominant contributions to the line shape that arise from power broadening, Doppler broadening, and laser line width have to be considered.

For the case of a two-level system, the power-broadened line width, $\Gamma_{\text {eff }}$, can be expressed in terms of the zero-power line width, $\Gamma_{0}$, as [23]

$\Gamma_{\text {eff }}=\Gamma_{0} \sqrt{1+I / I_{\mathrm{SAT}}}$

If the laser line width can be described by a Lorentzian with full-width half-maximum $\Gamma_{\mathrm{L}}$, the zero-power line width in (11) can be written as

$\Gamma_{0}=\Gamma+\Gamma_{\mathrm{L}}$

Because the line shape has a contribution due to Doppler broadening, it must be represented by a Voigt profile as described below. We can construct the Voigt profile by convolving (8) with a Doppler contribution so that

$\alpha(\omega, x)=K f_{i k} \Gamma_{\text {eff }} n_{0} \int_{-\infty}^{\infty} \exp \left[-2\left(\frac{x}{x_{0}}\right)^{2}\right] \frac{\exp \left\{-\left[2\left(\omega_{0}^{\prime}-\omega_{0}\right) / \Delta_{\mathrm{D}}\right]^{2}\right\}}{\left(\omega-\omega_{0}\right)^{2}+\left(\Gamma_{\text {eff }} / 2\right)^{2}} \mathrm{~d} \omega_{0}^{\prime}$

where $\omega_{0}^{\prime}$ is an integration variable, and $\Delta_{\mathrm{D}}$ is the Doppler width of the transition given by

$\Delta_{\mathrm{D}}=\frac{4 \pi u_{0}}{\lambda_{0}}$

The Doppler line width parameter, $\Delta_{\mathrm{D}}$, is defined by the most probable speed $u_{0}$ that characterizes the Maxwell-Boltzmann distribution of the cold gas and the resonant wavelength, $\lambda_{0}$.

Equation (14) can be expressed in closed form by performing a Taylor expansion of the integrand if the optical depth of the sample is small. This allows the absorption spectrum to be fit to a Voigt profile, if the Doppler contribution is known. In (15), $u_{0}$ is related to the sample temperature $T$ as

$u_{0}=\sqrt{\frac{2 k_{\mathrm{B}} T}{m_{\mathrm{Rb}}}}$

where, $k_{\mathrm{B}}$ is Boltzmann's constant and $m_{\mathrm{Rb}}$ is the ${ }^{85} \mathrm{Rb}$ mass. The most probable speed can be determined from the ballistic expansion of the atomic cloud as a function of time. The radius of the expanding cloud, $r(t)$, can be modelled by $[16,17]$

$r(t)=\sqrt{x_{0}^{2}+\left(u_{0} t\right)^{2}}$

where, $x_{0}$ is the initial radius of the cloud along the direction of the probe beam. Hence, the Doppler contribution to the line shape can be inferred from the expansion of the atomic cloud. 
Fig. 1. Schematic diagram illustrating the frequency chain used in the experiment; $\omega_{0}$ is the resonance frequency of the $F=3 \rightarrow F^{\prime}=4$ transition.

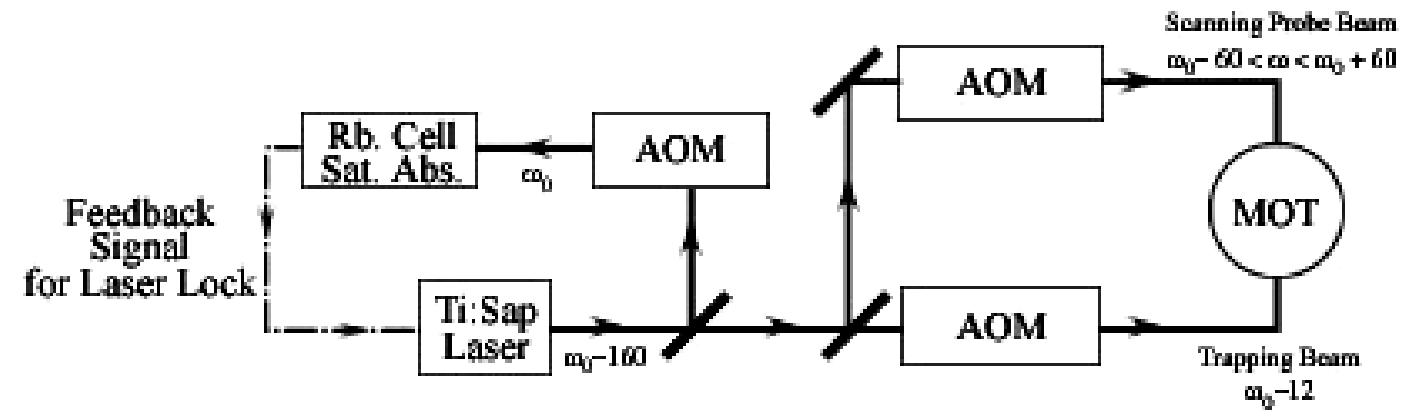

Fig. 2. Energy-level diagram illustrating the hyperfine structure in ${ }^{85} \mathrm{Rb}$.

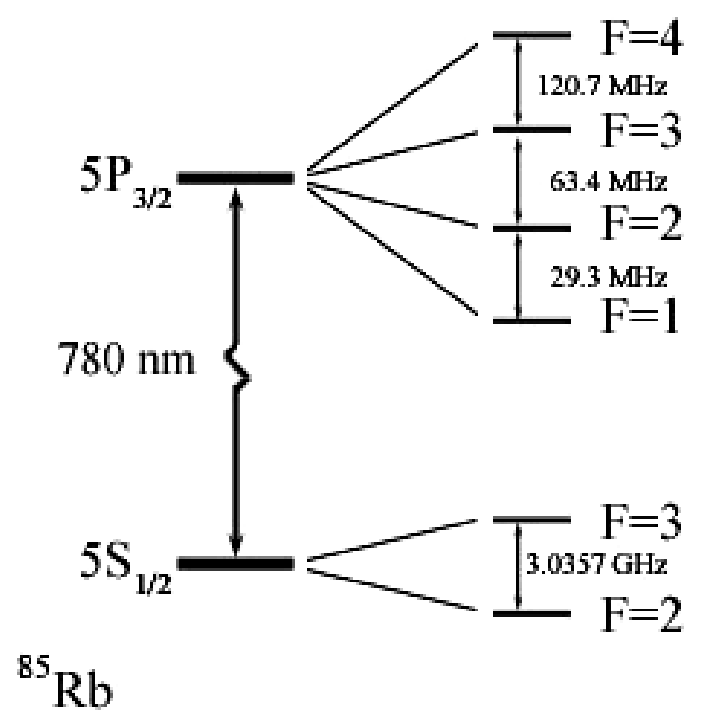

\section{Experimental details}

The three orthogonal trapping laser beams and the probe laser beam are derived from the same Ti:sapphire laser (Ti:Sap) using acousto-optic modulators (AOMs) (see Fig. 1).

The Ti:Sap is locked to an external Fabry-Perot cavity and has a line width of $\sim 2 \mathrm{MHz}$. The trapping laser beam and probe beam are generated using two AOMs operating in dual-pass configuration at $\sim 80 \mathrm{MHz}$ [24]. A weak beam split-off from the Ti:Sap is directed through another dual-pass AOM (operating at $\sim 80 \mathrm{MHz}$ ) and sent through a $\mathrm{Rb}$ vapour cell to obtain saturated absorption spectra. The laser is locked to the $F=3 \rightarrow F^{\prime}=4$ transition using frequency modulation spectroscopy (see Fig. 2).

Locking the laser involves modulating the RF oscillator used to modulate the $80 \mathrm{MHz}$ AOM used for saturated absorption at a frequency of $10 \mathrm{kHz}$ and feeding back the dispersion line shape to the laser. The repump beam is derived from a separate grating-stabilized diode laser with a line width of $\sim 1 \mathrm{MHz}$. This laser is locked using saturated absorption so that it is resonant with the $F=2 \rightarrow F^{\prime}=3$ transition. The intensity of this laser is controlled by an AOM operating at $\sim 40 \mathrm{MHz}$. 
Fig. 3. Typical dual-pass AOM setup; the angular position of the outgoing beam is insensitive to the AOM frequency since the distance between the lenses and the AOM is equal to the focal length.

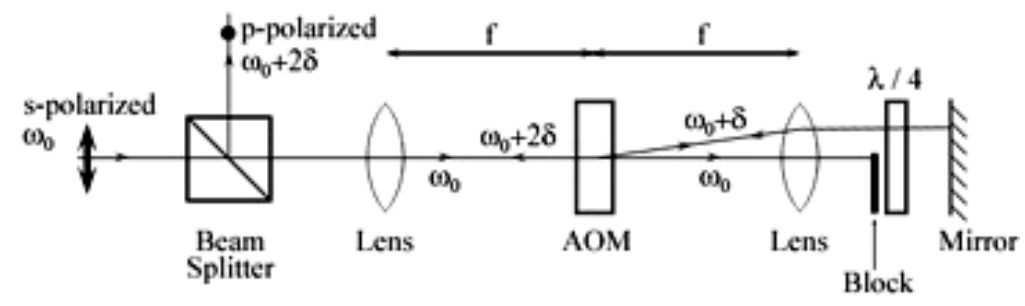

The dual-pass AOMs ensure that alignment of the laser beams is insensitive to the AOM operating frequency. Two lenses positioned at a distance of one focal length on either side of the AOM ensure that the twice diffracted beam returns along the direction of the incident beam. A polarizing beam splitter, quarter-wave plate and mirror are used to separate the incident and twice-diffracted laser beam. This configuration is shown in Fig. 3.

The magneto-optic trap is loaded from Doppler-broadened rubidium vapor. The background pressure in the vacuum chamber is $\sim 1 \times 10^{-9}$ Torr $\left(1\right.$ Torr $=133.322 \mathrm{~Pa}$ ). We typically accumulate $\sim 10^{8}$ atoms in $\sim 300 \mathrm{~ms}$ using (Gaussian) trapping beams with a $1 / e^{2}$ diameter of $32 \mathrm{~mm}$. The number of atoms is determined using a photomultiplyer tube (PMT) to measure the trap fluorescence. For typical experimental conditions, we measure the relative populations of the ground-state sublevels using absorption spectroscopy. As in ref. 13, we find that the atoms are predominantly in higher $|\mathrm{m}|$ states. Thus, it can be expected that the trap fluorescence will not be perfectly isotropic.

Immediately after turning off the trapping and repump beams in $\sim 1 \mu \mathrm{s}$ and the field gradient in $\sim 500 \mu \mathrm{s}$, the trapped atoms are optically pumped into the $\left(F=3, m_{\mathrm{f}}=3\right)$ state. Optical pumping is achieved by applying a weak $(\sim 1 \mathrm{G})$ quantization field along the probe direction by reversing the current in one of the anti-Helmholtz coils. Circularly polarized optical pumping beams (aligned to within a few degrees of the quantization axis and the direction of the probe beam) are turned on for $\sim 100 \mu \mathrm{s}$ in the presence of the magnetic field. These circularly polarized pulses are resonant with the $F=3 \rightarrow F^{\prime}=3$ and $F=3 \rightarrow F^{\prime}=2$ transitions.

Since these optical pumping beams are decoupled from the optically pumped $\left(F=3, m_{\mathrm{f}}=3\right.$ ) state, it should be possible to ensure that the entire sample is optically pumped. Another advantage of this technique is that unnecessary heating due to additional laser interaction is avoided after the atoms have been optically pumped [18]. To verify that optical pumping is complete, the $\left(F=3, m_{\mathrm{f}}=3\right) \rightarrow$ $\left(F^{\prime}=3, m_{\mathrm{f}}^{\prime}=3\right)$ laser is turned on after both pumping lasers are turned off and the fluorescence due to this pulse is recorded. The absence of fluorescence is used to indicate a complete population transfer to the $\left(F=3, m_{\mathrm{f}}=3\right)$ state. In our experiments, it was established that more than $95 \%$ of the atoms were optically pumped. Incomplete pumping is attributed to the angle between the optical pumping beams and the quantization field.

After optical pumping, a weak, circularly-polarized probe beam is pulsed on for $\sim 500 \mu \mathrm{s}$. The probe beam is turned on 1-2 ms after the optical pumping beams are turned off. While the probe beam is on, the frequency of the RF oscillator controlling the probe AOM is varied using a triangular ramp from a waveform generator with a $10 \mathrm{kHz}$ frequency. This ensures that the probe beam's frequency is scanned across $\sim 120 \mathrm{MHz}$ centered on the $F=3 \rightarrow F^{\prime}=4$ resonance line. Therefore, the probe beam's frequency varies at a rate of $2.4 \mathrm{kHz} / \mathrm{ns}$. The probe was Gaussian and its diameter is measured by scanning a rotating knife edge across its profile. The absorption signal is recorded on an oscilloscope using a Si-pin photodiode (rise time $<1 \mathrm{~ns}$ ) and a low-noise preamplifier. For typical preamplifier gain settings and scan conditions, we estimate the frequency resolution of our detection system to be $\sim 25 \mathrm{kHz}$. 
Fig. 4. Typical background subtracted absorption signal (continuous line) with fit given by (11) (broken line). From the fit $n_{0}=4.0 \times 10^{9} \mathrm{~cm}^{-3}, \Gamma_{\text {eff }}=8.362 \pm 0.019 \mathrm{MHz}$. Probe intensity $=0.480 \mathrm{~mW} / \mathrm{cm}^{2}, 1 / e^{2}$ diameter $=950 \mu \mathrm{m}, N=3.2 \times 10^{8}, \mathrm{~d} B / \mathrm{d} z=3.02 \mathrm{G} / \mathrm{cm}$.

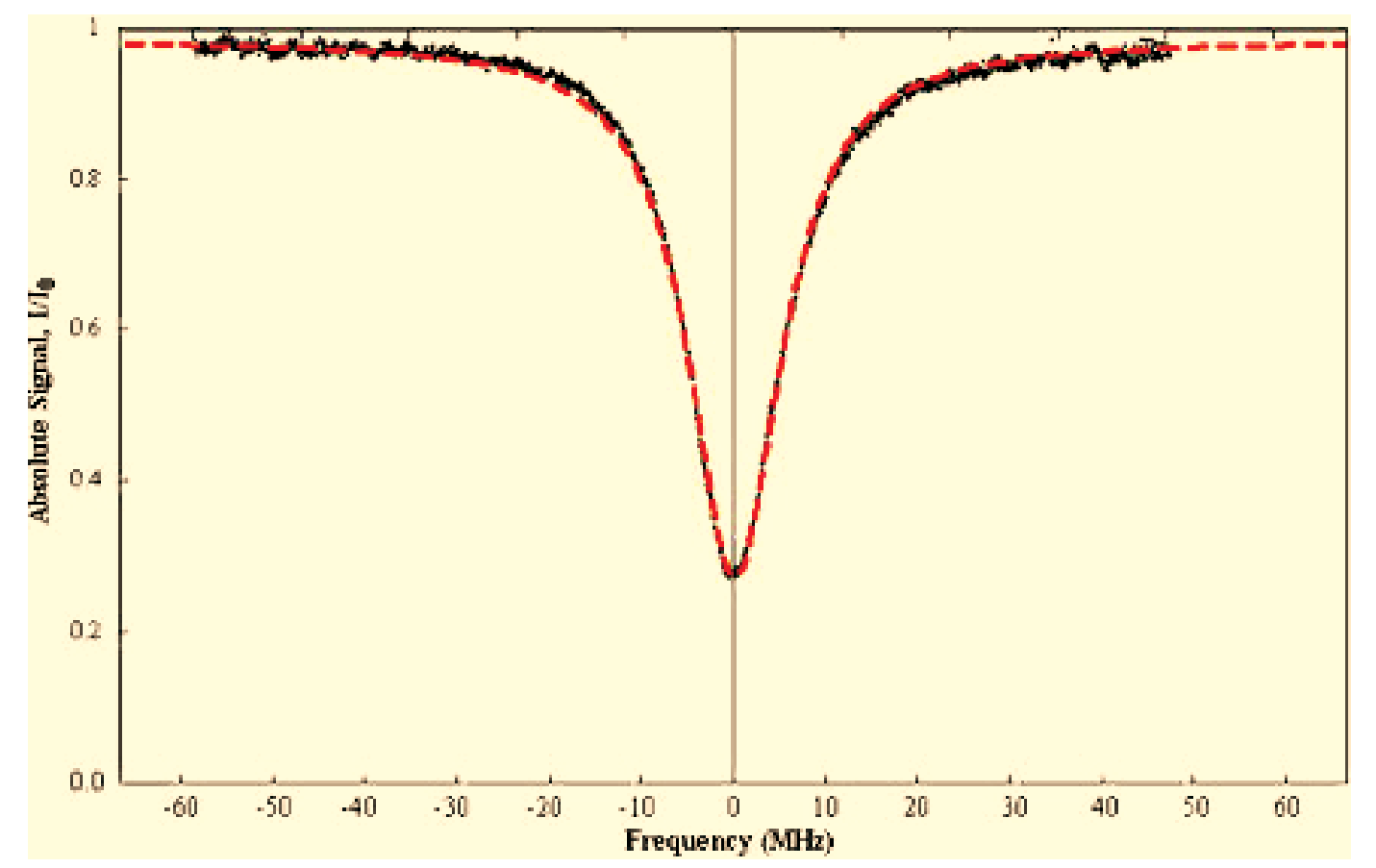

The light intensity recorded by the detector exhibits a characteristic variation as a function of the scan frequency due to the variation in the efficiency of the AOM cavity. In addition, there is a small DC contribution due to scattered light. The background signal due to variation in AOM efficiency is measured by recording the signal in the absence of the trapped sample. The signal due to all other DC contributions is measured by blocking the probe beam.

Two CCDs are used to measure the initial $1 / e^{2}$ diameter of the cloud along orthogonal directions. For our conditions, the CCD images resulted in Gaussian distributions so that the shape of the cloud can be described by (4). The CCDs are also used to record the expansion of the atomic cloud modelled by (17). This is achieved by turning off the confining forces, strobing on the trapping, and repumping beams (for $500 \mu \mathrm{s}$ ) after a variable time delay and recording the resulting fluorescence. This measurement allows us to measure the Doppler contribution to the natural line width.

\section{Results and discussion}

Figure 4 shows the typical absorption line shape as a function of scan frequency. The scan consists of $\sim 1900$ points and the line shape is determined by averaging 16 repetitions. This line shape is fit to the Lorenztian function described by (11) with the peak density $n_{0}$, resonant frequency $\omega_{0}$, and effective line width $\Gamma_{\text {eff }}$ as free parameters. The size of the cloud along the direction of the probe, $x_{0}$, is determined from the CCD measurement. The oscillator strength used in the fits for our two-level model is 0.338 as obtained from (10). The fit, shown as a broken line in Fig. 4, gives $\Gamma_{\text {eff }}=(8.362 \pm 0.019) \mathrm{MHz}$. We note that the fit is indistinguishable from the Lorentzian described by (11) and suggests that the technique is suitable for a precision measurement of the natural line width. We also note that the precision measurement would involve fits to the closed form solutions of the Voigt profile described by (14). 
Fig. 5. The broadening of the absorption line shape as a function of probe laser power. $1 / e^{2}$ diameter $=$ $4.5 \mathrm{~mm}$. From fit $I_{\mathrm{SAT}}=2.96 \pm 0.36 \mathrm{~mW} / \mathrm{cm}^{2} ; \Gamma_{0}=8.078 \pm 0.020 \mathrm{MHz}$.

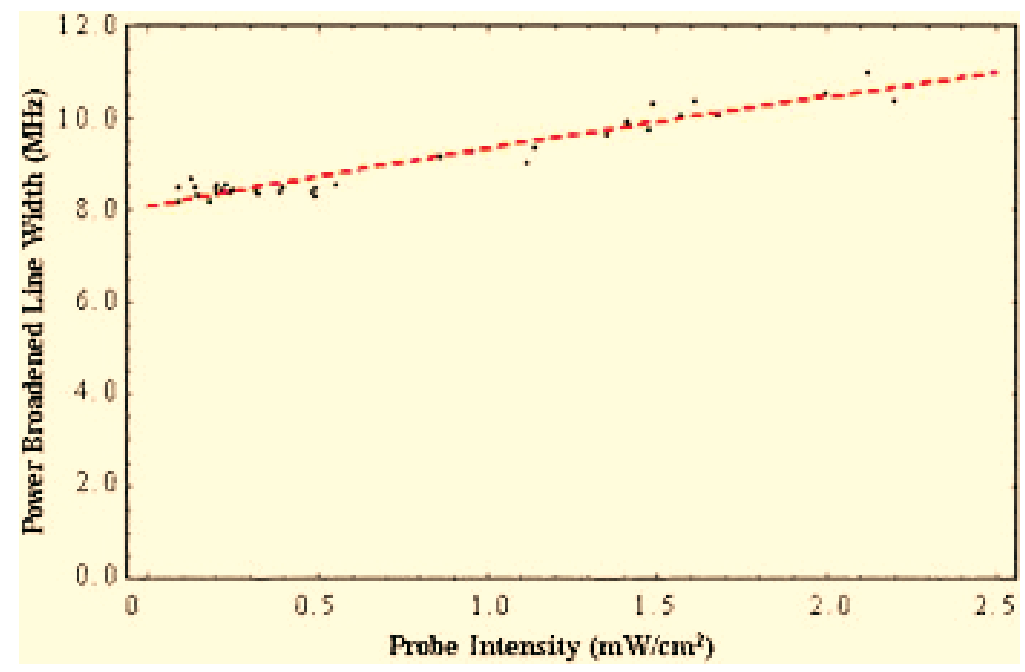

Table 1. Line width contributions and their associated uncertainties.

\begin{tabular}{llll}
\hline Contributor & Line width $(\mathrm{MHz})$ & Correction $(\mathrm{MHz})$ & Uncertainty $(\mathrm{MHz})$ \\
\hline $\begin{array}{l}\text { Line width measured from Fit, } \Gamma_{\text {eff }} \\
\quad \text { (see Fig. 4) }\end{array}$ & 8.362 & 0.019 \\
$\begin{array}{l}\text { Power-broadening correction } \\
\begin{array}{l}\text { Doppler contribution to Voigt } \\
\quad \text { profile }\end{array}\end{array}$ & -0.284 & 0.020 \\
$\begin{array}{l}\text { Resulting line width (including } \\
\text { undetermined laser line width } \\
\text { contribution) }\end{array}$ & 8.078 & -0.027 & $(<0.001)$ \\
\hline
\end{tabular}

Figure 5 shows the effect of varying the probe beam power on the line width of the absorption line shape. This effect is well understood [25] and has been previously studied in atomic systems [26, 27]. For each data point in Fig. 5, the probe power was obtained from a calibration curve that related the average signal amplitude during a frequency sweep to the absolute power measured by a silicon detector. The resulting data are fit to (12) and allows us to infer $I_{\mathrm{SAT}}=(2.96 \pm 0.36) \mathrm{mW} / \mathrm{cm}^{2}$. By extrapolating to zero probe intensity we obtain $\Gamma_{0}=(8.078 \pm 0.020) \mathrm{MHz}$. The theoretical value for $I_{\mathrm{SAT}}$ for the optically-pumped rubidium system is $3.24 \mathrm{~mW} / \mathrm{cm}^{2}$, which is in good agreement with the results. This comparison does not include additional systematic corrections for the probe spatial profile and power, which are estimated to be at the $5 \%$ level.

Table 1 summarizes the systematic contributions to the line width measurement addressed in this work. The correction due to power broadening was determined from the fit shown in Fig. 5. The line width obtained by extrapolating to zero power is tabulated in Table 1 . The overall uncertainty is $\sim 28 \mathrm{kHz}$, and is determined by summing these contributions in quadrature. This estimate ignores the resolution limit of $\sim 25 \mathrm{kHz}$ due to the detection system. This contribution can be made negligible by further reducing the scan rate $(\sim 100 \mathrm{~Hz})$ and using a larger bandwidth preamplifier.

The correction to the line width due to Doppler broadening can be determined by observing the ballistic expansion of the cloud (see Fig. 6) to determine the temperature and using the measurement 
Fig. 6. Ballistic expansion of atomic cloud; fit results in $u_{0}=18.0 \mathrm{~cm} / \mathrm{s}, x_{0}=1.34 \mathrm{~mm}$. Using (17), we find that $T=(166 \pm 3) \mu \mathrm{K}$.

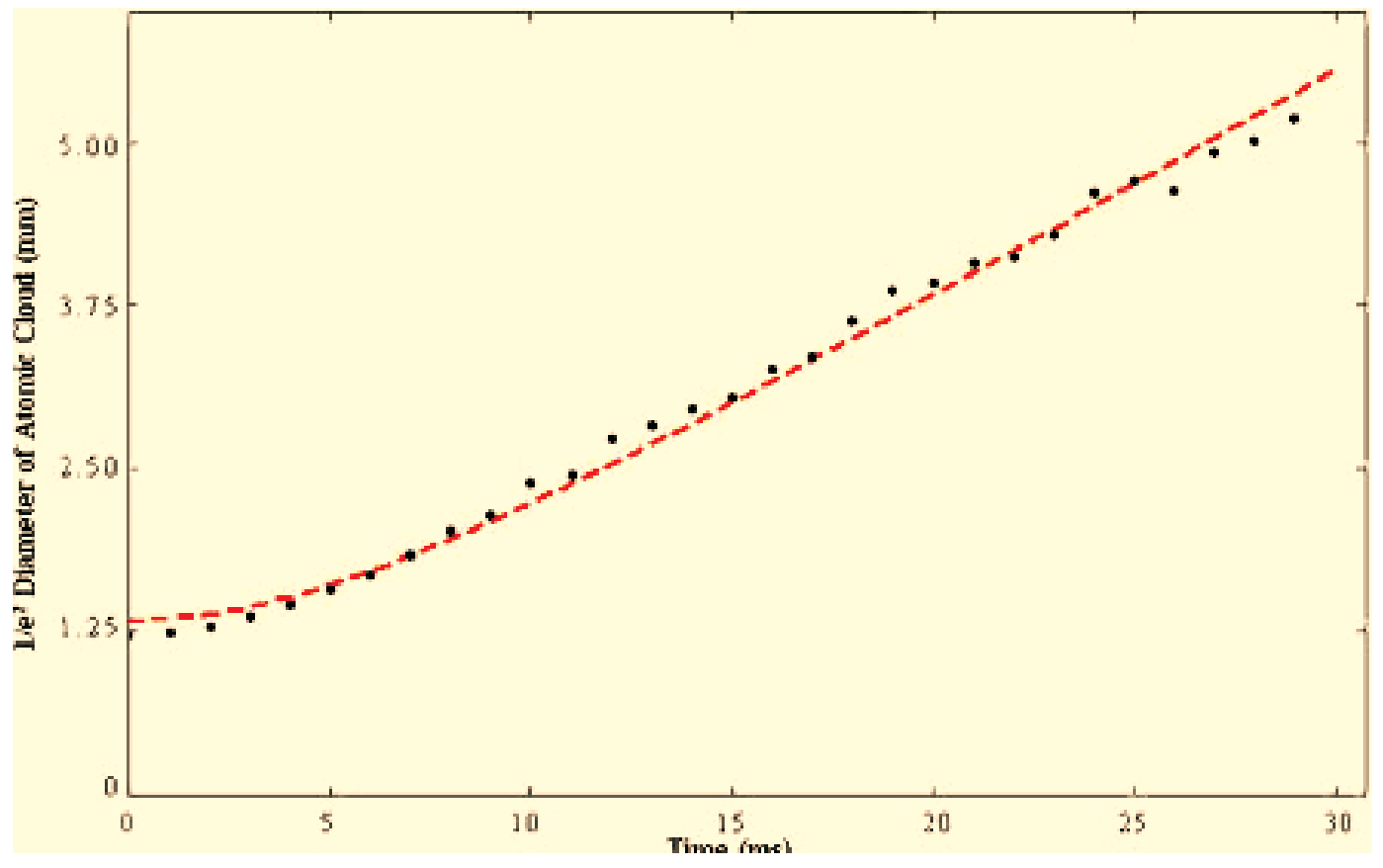

to model the Voigt profile. This correction is small compared with the effects of power broadening and laser line width and typically results in broadening at the $0.5 \%$ level for our sample. Because it is routinely possible to achieve temperatures of $15 \mu \mathrm{K}$ without significant atom loss, this contribution can be substantially reduced.

It is clear that the main challenge associated with the precision measurement of the natural line width is the measurement of the laser line width. The line width of the Ti:sapphire laser $(\sim 2 \mathrm{MHz})$ used in this experiment can be narrowed by improving the stability of the laser cavity and by improving the finesse and stability of the external cavity to which the laser is referenced. However, these measures are fairly extensive and difficult to achieve [7]. A more reasonable approach is to modify the electronic feed-back loops to reduce the line width to $200 \mathrm{kHz}$ (at the expense of the ability to scan over a wide range of frequencies). If a separate laser could be used for atom trapping and state preparation, then light from the modified Ti:Sap could be sent into a high-finesse Fabry-Perot cavity as in ref. 19 to make real-time line-width measurements with a precision of $\sim 10 \mathrm{kHz}$. A portion of the light from this laser could then be used for measuring absorption spectra. We anticipate that the natural line width could be determined to a precision of $\sim 50 \mathrm{kHz}$ based on the relative contributions of the main sources of error. Alternatively, a diode laser locked to an external cavity with a line width of $\sim 15 \mathrm{kHz}$ [28] could be used as the probe.

In a precision measurement, the absorption spectrum could be recorded as a function of probe intensity for optical depths much less than unity so that the line shape can be fit to the closed-form expression for the Voigt profile. The effective line width can be extrapolated to zero intensity. This measurement can be repeated as a function of density so that the line width can be corrected for the opacity of the sample as in ref. 7. Additional corrections for laser line width can be applied to obtain the natural line width. It will also be necessary to correct for the effect of probe-induced atomic recoil on the line shape [29] as well as optical pumping during the period of the scan. Both these effects are 
Fig. 7. Comparison of the absolute density of the $\left(F=3, m_{\mathrm{f}}=3\right)$ population obtained using two separate techniques; triangles represent the density obtained using the dimensions of the cloud and the number of atoms measured by the PMT; circles represent the density based on the absorption technique. $1 / e^{2}$ diameter $=950 \mu \mathrm{m}$.

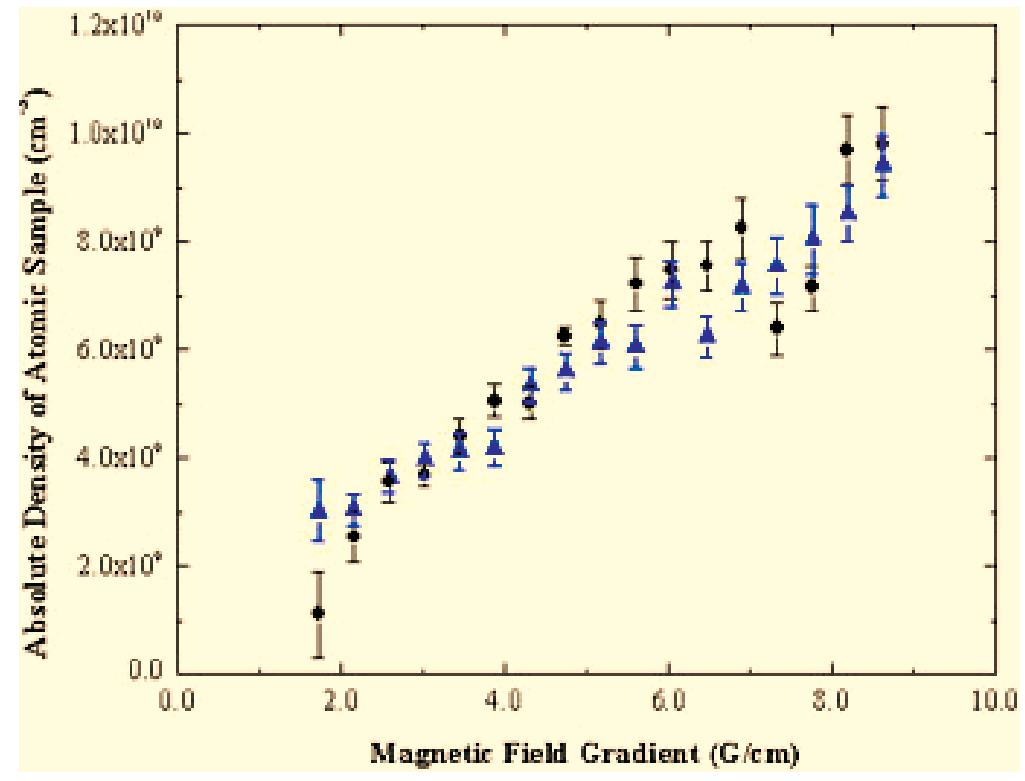

known to produce asymmetrical line shapes and can result in corrections at the level of a few kilohertz. It would also be necessary to increase the degree of optical pumping so that additional corrections such as differential AC Stark shifts and broadening due to residual ambient magnetic fields are negligible. Other proposed improvements include improving the spatial profile of the probe using a fibre coupler. The signal-to-noise ratio in our power measurements would also need to be improved by a factor of $\sim 5$ to achieve the same measurement uncertainty as in ref. 7.

We now discuss the comparison of the absolute density determined from the absorption spectra of the two-level atoms and the density determined from the fluorescence. The absorption spectra are obtained after optically pumping the system, whereas the fluorescence is measured with the atoms predominantly in the higher $|m|$ states. The fluorescence measurement relies on determining the power in the trapping beams and our results have been corrected for small optical losses that accumulate over the beam paths. The density of the sample was varied by changing the magnetic field gradient. Figure 7 compares measurements based on the two techniques. For our conditions, the scaling laws that govern the density are strongly affected by radiation trapping. As the field gradient is increased, the density of the sample is expected to increase and then saturate. As the magnetic field gradient becomes stronger, the number of atoms also increases and then saturation begins. We note that the data from both techniques agree with these expectations. We conclude that the agreement between the two techniques is satisfactory over the range of field gradients. Corrections to the absorption measurement due to incomplete optical pumping and to the fluorescence measurement due to anisotropy in the fluorescence are difficult to quantify and have not been included.

\section{Conclusions}

In summary, we have presented a comparison of density measurements of a sample of two-level trapped atoms using two techniques. These techniques have been useful for obtaining reliable estimates 
of trap conditions and for obtaining reproducible conditions for on-going experiments in atom interferometry. The quality of the absorption spectra and a study of the dominant systematic effects suggest that a precision measurement of the natural line width can be carried out by measuring the laser line width during the experiment. The required resolution can be obtained using a high-finesse Fabry-Perot cavity. Advantages of this technique include reduced experimental complexity and the ability to extend the measurement to other transitions in ${ }^{85} \mathrm{Rb}$ and ${ }^{87} \mathrm{Rb}$. Such measurements would allow detailed comparisons with calculations of the structure of multilevel atoms and permit tests of predictions for isotopic differences.

\section{Acknowledgements}

This work was supported by the Canada Foundation for Innovation, Ontario Innovation Trust, the Natural Sciences and Engineering Research Council of Canada, York University, and Photonics Research Ontario.

\section{References}

1. E.L. Raab, M. Prentiss, A. Cable, S. Chu, and D.E. Prichard. Phys. Rev. Lett. 59, 2631 (1987).

2. C. Monroe, W. Swann, H. Robinson, and C. Wieman. Phys. Rev. Lett. 65, 1571 (1990).

3. A. Peters, K.Y. Chung, and S. Chu. Nature (London), 400, 849 (1999) and references therein.

4. T.L. Gustafson, P. Bouyer, and M.A. Kasevich. Phys. Rev. Lett. 78, 2046 (1997).

5. D.S. Weiss, B.C. Young, and S. Chu. Phys. Rev. Lett. 70, 2706 (1993).

6. Th. Kisters, K. Zeiske, F. Riehle, and J. Hemcke. Appl. Phys. B, 59, 89 (1994).

7. C.W. Oates, K.R. Vogel, and J.L. Hall. Phys. Rev. Lett. 76, 2866 (1996).

8. W.I. McAlexander, E.R.I. Abraham, and R.G. Hulet. Phys. Rev. A, 54, R5 (1996).

9. K.M. Jones, P.S. Julienne, P.D. Lett, W.D. Phillips, E. Tiesinga, and C.J. Williams. Europhys. Lett. 35, 85 (1996).

10. R.F. Gutterres, C. Amiot, A. Fioretti, C. Gabbanini, M. Mazzoni, and O. Dulieu. Phys. Rev. A, 66, 024502 (2002).

11. G. Santarelli, Ph. Laurent, P. Lemonde, A. Clairon, A.G. Mann, S. Chang, A.N. Luiten, and C. Salomon. Phys. Rev. Lett. 82, 4619 (1999).

12. C. Fertig and K. Gibble. Phys. Rev. Lett. 85, 1622 (2000) and references therein.

13. C.G. Townsend, N.H. Edwards, C.J. Cooper, K.P. Zetie, C.J. Foot, A.M. Steane, P. Szriftgiser, H. Perrin, and J. Dalibard. Phys. Rev. A, 52, 1423 (1995).

14. C.J. Cooper, G. Hillenbrand, J. Rink, C.G. Townsend, K. Zetie, and C.J. Foot. Europhys. Lett, 28, 397 (1994).

15. K. Lindquist, M. Stephens, and C. Wieman. Phys. Rev. A, 46, 4082 (1992).

16. D.S. Weiss, E. Riis, Y. Shevy, P.J. Ungar, and S. Chu. JOSA B, 6, 2072 (1989).

17. D. Guery-Odelin, J. Soeding, P. Desbiolles, and J. Dalibard. Opt. Exp. 2, 323 (1998).

18. K.E. Gibble, S. Kasapi, and S. Chu. Opt. Lett. 17, 526 (1992).

19. K.S. Repasky, L.E. Watson, and J.L. Carlsten. Appl. Opt. 34, 2615 (1995).

20. J.E. Simsarian, L.A. Orozco, G.D. Sprouse, and W.Z. Zhao. Phys. Rev. A, 57, 2448 (1998).

21. U. Volz and H. Schmoranzer. Phys. Scr. T, 65, 48 (1996).

22. A. Gallagher and E.L. Lewis. Phys. Rev. A, 10, 231 (1974).

23. A. Corney. Atomic and Laser Spectroscopy, Oxford. 1977.

24. G. Spirou, I. Yavin, M. Weel, A. Vorozcovs, A. Kumarakrishnan, P.R. Battle, and R.C. Swanson. Can. J. Phys. 81, 625 (2003).

25. W. Demtröder. Laser spectroscopy. Springer-Verlag, Berlin. 1981.

26. P.G. Pappas, M.M. Burns, D.D. Hinshelwood, M.S. Feld, and D.E. Murnick. Phys. Rev. A, 21, 1955 (1980).

27. M.L. Citron, H.R. Gray, C.W. Gabel, and C.R. Stroud, Jr. Phys. Rev. A, 16, 1507 (1977).

28. T.P. Dinneen, C.D. Wallace, and P.L. Gould. Opt. Commun. 92, 277 (1992).

29. J.F. Lam and P.R. Berman. Phys. Rev. A, 14, 1683 (1976). 